\title{
Patient preference and satisfaction in erectile dysfunction therapy: a comparison of the three phosphodiesterase- 5 inhibitors sildenafil, vardenafil and tadalafil
}

\author{
Amr Abdel Raheem' \\ Philip Kell ${ }^{2}$ \\ 'St. Peter's Andrology Department, \\ The Institute of Urology, London, \\ and Cairo University, Egypt; ${ }^{2}$ St. Peter's \\ Andrology Department, The Institute \\ of Urology, London, UK
}

\begin{abstract}
Erectile dysfunction (ED) is a problem that may affect up to $52 \%$ of men between the ages of 40 and 70. It can be distressing because of its negative effect on self-esteem, quality of life, and interpersonal relationships. Oral phosphodiesterase-5 inhibitors (PDE5 inhibitors) are now the first choice of treatment in ED. The availability of three (sildenafil citrate, tadalafil, and vardenafil) well tolerated and effective oral PDE5 inhibitors gives treatment options for men with ED. Although the mechanism of action is the same for the three drugs, they differ in their pharmacokinetics. Several preference studies were conducted between the three PDE5 inhibitors but they were not free from bias. Because of the lack of overwhelming reliable data showing that one PDE5 inhibitor is superior to another, current opinion is that the individual patient should have the opportunity to test all three drugs and then select the one that best suits him and his partner.
\end{abstract}

Keywords: erectile dysfunction, PDE5 inhibitors, patient preference

\section{Introduction}

Erectile dysfunction (ED) is defined as the consistent or recurrent inability to attain and/or maintain an erection sufficient for sexual performance. ${ }^{1}$ ED has an overall prevalence of $52 \%$ in men between the ages of 40 and 70 . Of these $52 \%, 17.2 \%$ have minimal ED, $25.2 \%$ have moderate ED while $9.6 \%$ have severe ED. ${ }^{2}$ The incidence of ED increases with age; however, it is important to remember that it is not an inevitable consequence of aging and that advancing age does not preclude sexual interest. With increasing life expectancy, it is anticipated that the prevalence of ED will rise. ${ }^{3}$

ED can be distressing because of its negative effect on self-esteem, quality of life, and interpersonal relationships. Erectile function is a key quality of life indicator for many men. Men with ED may endure diminished self-image and self-esteem, anxiety and fears of rejection, and even depression. ${ }^{4,5}$ The female partner may also suffer from female sexual dysfunction secondary to her partner's ED. ${ }^{6}$

The etiology of erectile dysfunction can be divided into psychological and physical factors although in most cases both mechanisms interplay. Psychological factors include depression, relationship issues, sexual ignorance, fear of failure, performance anxiety, and childhood or adult sexual abuse. Physical or organic ED may be vasculogenic as in diabetes, hypertension, hypercholesterolaemia and smoking, neurogenic as in spinal injuries, endocrinal as in hypogonadism and hypothyroidism, and local penile tissue factors as in Peyronie's disease. Recent studies have shown the association of ED with metabolic syndrome and cardiovascular disease. ED is now considered a marker for these conditions and consequently the management of ED now concentrates
St. Peter's Andrology Department,

The Institute of Urology, London, UK

Email pdkell@hotmail.com 
on screening for, and preventing, cardiovascular diseases as well as treating the ED itself. ${ }^{7}$

Oral phosphodiesterase-5 inhibitors (PDE5 inhibitors) are now the first choice of treatment in ED due to their efficacy and safety and the fact that they are non invasive. ${ }^{8}$

In the era of PDE5 inhibitors the treatment of ED can be simple, effective and with good response rates. Other lines of therapy such as intracorporal or penile implants are resorted to in patients who fail to respond to PDE5 inhibitors. The patientoriented approach for diagnosis and treatment of ED is the preferred approach for the majority of men. This means that medical history, psychosexual history, physical examination and basic laboratory investigations are done for all patients while other investigatory modalities are reserved for selected cases. ${ }^{9}$ Following the initial assessment patients should have the available treatment options explained, including the benefits and the potential complications of each modality. The treatment plan is thus formulated jointly between the physician and patient. Where possible, the patient's partner should also be involved, taking into consideration the patient's preferences, concerns and expectations. ${ }^{10,11}$ This allows the formulation of a treatment plan which can be tailored to the needs and lifestyle of an individual patient.

The availability of three well tolerated and effective oral PDE5 inhibitors gives treatment options for men with ED. A patient's preference for an ED therapy is determined mainly by its efficacy, tolerability and safety. However other factors are also involved and they depend on the patient and his partner's expectations from the treatment and its overall effect on the quality of their sex life.

\section{Mechanism of action of PDE5 inhibitors}

Erection is a hemodynamic phenomenon mediated via the nitric oxide (NO)-cyclic guanosinemonophosphate (cGMP) pathway. Following sexual stimulation, neuronal impulses cause the release of NO into the corpora cavernosa. cGMP acts as a second messenger for NO, causing smooth muscle relaxation leading to increased arterial blood flow and sinusoidal relaxation which in turn causes compression of the veins preventing venous return, and erection occurs. Phosphodiesterase PDE5 is an enzyme that breaks down cGMP. PDE5 inhibitors act as competitive inhibitors of PDE5 thus helping erection. ${ }^{12}$

\section{Pharmacokinetics of the three PDE5 inhibitors}

As competitive inhibitors of PDE5, PDE5 inhibitors have structures derived from cGMP. Sildenafil and vardenafil have very similar molecular structures. In contrast, tadalafil has a different chemical structure from sildenafil and vardenafil. These structural differences lead to differences in the pharmacokinetics of the three drugs. ${ }^{13}$

The mean time to maximum plasma concentration of sildenafil and vardenafil is $1 \mathrm{~h}$ and for tadalafil is $2 \mathrm{~h}$, while the half-lives of sildenafil and vardenafil are $4 \mathrm{~h}$ and that of tadalafil is $17.5 \mathrm{~h}$. Food high in fat delays and reduces the absorption of sildenafil and vardenafil, but does not affect the rate or extent of absorption of tadalafil. ${ }^{15}$ These pharmacokinetics give tadalafil the advantage of allowing spontaneity in sex and giving the couple an open window for successful intercourse, making it different from the other two PDE5 inhibitors.

\section{Drug interactions of the three PDE5 inhibitors}

All PDE5 inhibitors act via the NO/cGMP mechanism thus they can have synergistic effects when used with nitrates or NO donors causing sever hypotension. All PDE5 inhibitors are broken down mainly via cytochrome P450 thus a dose adjustment should be considered when given in combination with cytochrome P450 inhibitors like HIV protease inhibitors, erythromycin and ketoconazole as these drugs may inhibit PDE5 inhibitor plasma clearance and potentiate its effect. ${ }^{16}$

\section{Efficacy of the three PDE5 inhibitors}

All three PDE5 inhibitors have been shown to be effective and safe in the broad population of men with ED as well as

Table I Pharmacokinetics of the three PDE5 inhibitors ${ }^{14}$

\begin{tabular}{llllll}
\hline Drug & $\mathbf{T}_{\max }(\mathbf{h})^{\mathbf{a}}$ & $\begin{array}{l}\text { Earliest onset } \\
\text { of action }(\mathbf{m i n})\end{array}$ & $\mathbf{T}_{1 / 2}(\mathbf{h})^{\mathbf{b}}$ & Duration of effect (h) & $\begin{array}{l}\text { Recommended dosing time (h) } \\
\text { prior to intercourse }\end{array}$ \\
\hline Sildenafil $100 \mathrm{mg}$ & $\mathrm{I}$ & 30 & 4 & $4-6$ (up to I2) & I \\
Tadalafil $20 \mathrm{mg}$ & 2 & 20 & 17.5 & $24-36$ (up to72) & $0.5-12$ \\
Vardenafil $20 \mathrm{mg}$ & 0.7 & 10 & 4.5 & $5-7$ (up to 12) & $0.5-1$ \\
\hline
\end{tabular}

${ }^{\mathrm{a}} \mathrm{T}_{\max }$ time needed to reach maximum plasma concentration $\left(\mathrm{C}_{\max }\right)$, which corresponds to onset of action.

${ }^{\mathrm{b}} \mathrm{T}_{1 / 2}{ }^{\max }$ half-life, which corresponds to duration of efficacy. 
different subpopulations. ${ }^{17}$ Patients who fail to respond to one PDE5 inhibitor may respond to other PDE5 inhibitors. ${ }^{18}$ Failure on a PDE5 inhibitor should not be considered before at least 8 failures to respond to maximum dose medications. ${ }^{19,20}$ Daily dosing with PDE5 inhibitors for several months in previous PDE5 inhibitor non-responders may rescue more than $50 \%$ of patients. ${ }^{21,22}$ Treatment of concomitant diseases, eg, diabetes, hyperlipidemia and treatment of hypogonadism can improve erectile function and response to PDE5 inhibitors. $^{23-25}$

\section{Side effects of the three PDE5 inhibitors}

Side effects occur due to vasodilatation elsewhere in the body caused by inhibition of other phosphodiesterase enzymes. Side effects are similar for the three drugs and include headache, flush, dyspepsia and rhinitis, with the exception of visual disturbances which occur with sildenafil and back pain which occurs with tadalafil. Generally these side effects are mild to moderate and resulted in $1 \%-2 \%$ drop outs in clinical trials. ${ }^{17}$

\section{Precautions and contraindications of PDE5 inhibitors}

Because PDE5 inhibitors act in a similar way via the $\mathrm{NO}$ / cGMP mechanism, the contraindications are similar for the three drugs. Patients with poor general condition, eg, severe cardiovascular disease, for whom sexual activity may be risky should not be given ED treatment. In patients receiving nitrate or NO-donors, treatment with PDE5 inhibitors is contraindicated because they act via the same NO/cGMP mechanism and concomitant treatment with a PDE5 inhibitor can lead to severe hypotension and shock. ${ }^{26}$

Howeversome of the patients with stable coronary heart disease and erectile dysfunction currently taking oral longacting nitrates can be withdrawn from this treatment without facing disadvantages and can then be successfully treated with a PDE5 inhibitor. ${ }^{27}$

Table 2 Side effects of PDE5 inhibitors ${ }^{17}$

\begin{tabular}{llll}
\hline Side effect & Sildenafil & Vardenafil & Tadalafil \\
\hline Headache & $14.6 \%$ & $14.5 \%$ & $14 \%$ \\
Flush & $14.1 \%$ & $11.1 \%$ & $4 \%$ \\
Dyspepsia & $6.2 \%$ & $3.7 \%$ & $10 \%$ \\
Rhinitis & $2.6 \%$ & $9.2 \%$ & $5 \%$ \\
Back pain & $0 \%$ & $0 \%$ & $6 \%$ \\
Visual disturbances & $5.2 \%$ & $0 \%$ & $0 \%$ \\
\hline
\end{tabular}

Rare reports of sudden loss of vision due to non-arteritic anterior ischemic optic neuropathy (NAION) led the FDA to update the labels of all three products. The labels now advise doctors to stop PDE5 inhibitor therapy in the event of sudden loss of vision in one or both eyes and to discuss the increased risk of NAION in patients who have already experienced NAION. At present, however, it is not possible to determine whether these events were related to PDE5 inhibitors or to other factors. ${ }^{15}$ Conditions predisposing to NAION are: age $>50$, heart disease, diabetes, hypertension, high cholesterol, smoking and certain eye problems. ${ }^{17}$

There are certain precautions when using PDE5 inhibitors in patients taking $\alpha$-blockers due to the possibility of symptomatic hypotension with concomitant use. Sildenafil, vardenafil and tadalafil could be used with caution only in patients stabilised on the $\alpha$-blocker therapy. Furthermore the PDE5 inhibitors should be initiated at the lowest recommended dose. ${ }^{28-30}$ However one study showed that tadalafil had no clinically significant effect on blood pressure changes caused by tamsulosin. ${ }^{31}$

\section{Patient preference}

Clinical decision making is based on many factors. Some clinicians will claim to rely solely on research evidence, others their own clinical experience, but will consciously or at least subconsciously be affected by their own value systems. The availability of three, well tolerated and effective oral PDE5 inhibitors gives physicians treatment options for men with ED. The treatment of ED is unlike most other fields of medicine in that the outcome of treatment is not based on a laboratory result and cannot be measured by radiology, but

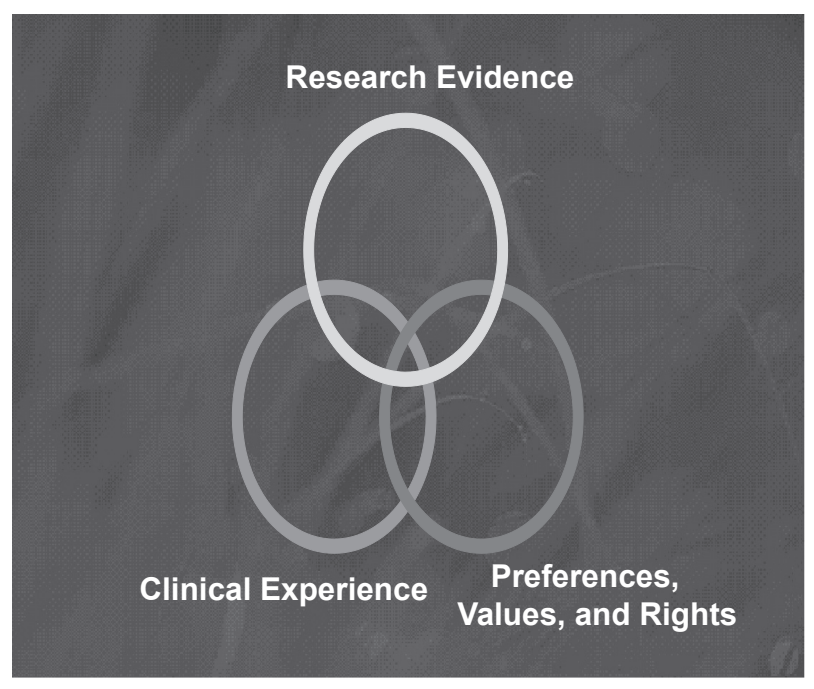

Figure I How decisions are made in clinical practice. 
is based solely on the judgment of the patient. Hence the "best" treatment for patients can really be judged only by the patients themselves and their partners. Some of the issues that affect choice are listed below.

Following the release of vardenafil and tadalafil several preference studies were conducted for the three PDE5 inhibitors. Many of these studies showed that patients preferred tadalafil. However these studies were criticized for their design and contradictory results. Studies from the industry tend to show preference for their own drug, whereas independent studies tend to show no major difference in preference. ${ }^{32}$

In a double-blind crossover study between tadalafil $20 \mathrm{mg}$ and sildenafil at a flexible dose, of the 181 who completed the study, $73 \%$ preferred tadalafil for the extension period. ${ }^{33}$

An open-label, one-way crossover trial involved patients on sildenafil and consisted of a 1-week screening phase, a 3-week sildenafil assessment phase, a 1-week washout phase, a 6-week tadalafil initiation phase, a 3-week tadalafil assessment phase, and a 6-month extension phase, during which patients received their treatment of choice free of charge. Of the 147 men who completed the trial, 133 (90.5\%) chose tadalafil and $14(9.5 \%)$ chose sildenafil. ${ }^{34}$

In another open-label multicenter study, men who were taking sildenafil at doses of 25-100 mg tested tadalafil $20 \mathrm{mg}$ for 8 weeks. Of the 2453 men, $82 \%$ preferred tadalafil. The main factors influencing this choice were an improvement in timing concerns and in sexual self confidence. Patients who took $>2.5$ sildenafil doses per week or high doses of sildenafil had a greater likelihood of remaining on sildenafil. ${ }^{35}$

Similarly, in a recent open-label European multicenter preference study between sildenafil and tadalafil involving

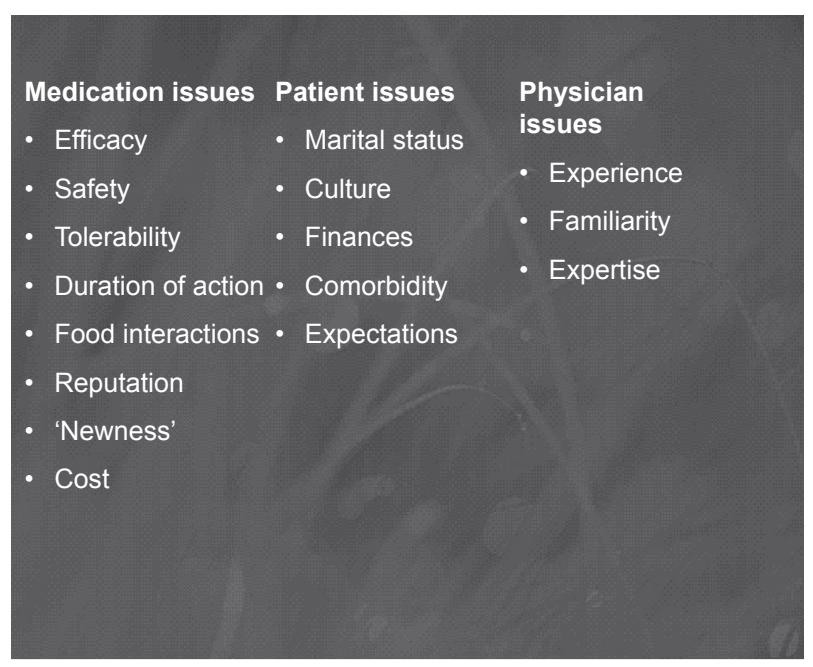

Figure 2 Factors affecting preference for a PDE5 inhibitor. patients naïve to PDE5 inhibitor therapy, patients were randomized to receive sildenafil for 12 weeks followed by tadalafil for 12 weeks or vice versa. Each 12-week period included an 8-week dose optimization phase and a 4-week assessment phase and 7-10 days' washout. Patients completing the study then got to choose which treatment to continue on for an 8-week extension phase. A total of 294 men completed the study; $29 \%(n=85)$ chose sildenafil for the extension phase and $71 \%(n=206)$ chose tadalafil. The patient's preference was not affected by treatment sequence, ED severity or etiology, age, or dosage. Both drugs were found effective and safe. ${ }^{36}$

In contrast, in an independent study involving PDE5naïve patients, 418 patients tested each PDE5 inhibitor at least 4 times. There was no difference in patient preference or in the drug-induced international index of erectile function score increase between the three drugs. However, young men with mild dysfunction preferred tadalafil, while older men, with more severe ED, preferred sildenafil or vardenafil. ${ }^{37}$

Other studies tried to detect patient preference indirectly by monitoring prescription data. An analysis of prescription data from 40,000 pharmacies in the US showed that men who are prescribed sildenafil as an initial treatment for ED are less likely to switch to another PDE5 inhibitor than those who are initially prescribed vardenafil or tadalafil..$^{38}$ Another study from the UK analyzed the prescription data of 2703 patients who initiated treatment for ED with a PDE5 inhibitor. Results showed that patients who were initially prescribed sildenafil are less likely to switch to another PDE5 inhibitor than those who were initially prescribed tadalafil or vardenafil. Patients who were initially prescribed tadalafil or vardenafil were nearly 4 times more likely to switch PDE5 inhibitors than patients who were initially prescribed sildenafil. ${ }^{39}$

In an evaluation of preference studies conducted for PDE5 inhibitors, Mulhal and Montorsi ${ }^{40}$ concluded that the studies had design defects which cause bias, sources of which included: not using a randomized, double-blind, crossover design, period effect (which involves adaptation to treatment so that the results will be in favor of the second drug, and may also occur due to unequal treatment periods), and carryover effect (the effect of the first treatment used may carry over to the next treatment, making the second treatment seem more effective than the first; it is a strong element of bias in trials where a single drug sequence is used). Other factors include the amount of time allotted for each medication, the amount of study drug, the dose of study drug, the instructions that accompany each drug (which all have to be 
the same for the studied drugs to avoid bias); sufficient time should be left between drugs for washout of any previous period or carryover effects. The selection criteria were also a source of bias in some trials in which vardenafil and tadalafil patients who had been previously treated successfully with sildenafil were included, but non responders or patients who had experienced severe side effects were excluded. This bias increased efficacy and decreased side effects for these drugs. Moreover, drug preference assessments should not wait until the end of the study and should not favor a particular drug. A questionnaire that covers efficacy, safety, and reasons for preference should be used. All preference data should be analyzed using the intent-to-treat group; for example, if discontinuations are the result of a treatment effect, analyses that include only study completers will overestimate treatment preference.

A well-designed crossover trial that assesses safety, efficacy and patient preference for all three PDE5 inhibitors has not yet been reported. ${ }^{39}$ Because of the lack of overwhelmingly reliable data showing that one PDE5 inhibitor is superior to another, current opinion is that the individual patient should have the opportunity to test all three drugs and then select the one that best suits him and his partner. ${ }^{32}$

This opinion was supported by an independent Swedish study ${ }^{14}$ in which patients were offered the opportunity to try the maximum dose of 8 short-acting tablets (4 tablets $100 \mathrm{mg}$ sildenafil and 4 tablets $20 \mathrm{mg}$ vardenafil) and 8 long-acting tablets ( $20 \mathrm{mg}$ tadalafil). Among the 138 patients who responded to treatment and completed the trial there was no significant difference between those who preferred a long-acting drug (tadalafil, 55\%) and those who preferred a short-acting drug (44\%: sildenafil, 27\%; vardenafil, 17\%). Moreover $19 \%$ of the patients requested a combination of both short- and long-acting drugs to accommodate their needs. Naïve patients preferred a short-acting drug while previously treated patients preferred a long-acting drug. Patients with mild ED tended to prefer a long-acting drug, but there was no difference in preference related to age or cause of ED. For patients preferring a short-acting drug, the primary reason was efficacy (82\%), few side effects $(15 \%)$ and rapid onset (3\%). For patients preferring a long acting drug, the primary reason was the long duration of effect $(96 \%)$ and few side effects $(4 \%)$.

\section{Conclusion}

Since the introduction of sildenafil citrate, PDE5 inhibitors have become the first line treatment for ED owing to their efficacy, safety and the fact that they are non invasive compared with other treatments. Following the introduction of tadalafil and vardenafil several patient preference studies were conducted; however, because they were not free from bias there are insufficient data for the clinician or the patient to judge which PDE5 inhibitor is superior. A good regimen is to allow the patient try all three PDE5 inhibitors and choose which best suits him and his partner. This regimen will also result in a higher success rate to PDE5 inhibitor therapy. Moreover some patients may prefer a combination of shortand long-acting PDE5 inhibitors.

\section{Disclosures}

The authors have no conflicts of interest to disclose.

\section{References}

1. NIH Consensus Conference. Impotence. NIH Consensus Development Panel on Impotence. JAMA. 1993;270(1):83-90.

2. Feldman HA, Goldstein I, Hatzichristou DG, Krane RJ, McKinlay JB. Impotence and its medical and psychosocial correlates: results of the Massachusetts Male Aging Study. J Urol. 1994;151(1):54-61.

3. Wagner G, Fugl-Meyer KS, Fugl-Meyer AR. Impact of erectile dysfunction on quality of life: patient and partner perspectives. Int J Impot Res. 2000;4(S1):44-46.

4. Sanchez-Cruz JJ, Cabrera-Leon A, Martin-Morales A, Fernandez A, Burgos R, Rejas J. Male erectile dysfunction and health-related quality of life. Eur Urol. 2003;44:245-253.

5. Fugl-Meyer AR, Lodnert G, Branholm IB, Fugl-Meyer KS. On life satisfaction in male erectile dysfunction. Int $J$ Impot Res. 1997;9:141-148.

6. Fisher WA, Rosen RC, Eardly I, Sand M, Goldstein I. Sexual experience of female partners of men with erectile dysfunction. J Sex Med. 2005;2(5):675-684.

7. Ponholzer A, Temml C, Obermayr R, Wehrberger C, Madersbacher S. Is erectile dysfunction an indicator for increased risk of coronary heart disease and stroke? 1. Eur Urol. 2005;48(3):512-518.

8. Briganti A, Salonia A, Gallina A, et al. Drug Insight: oral phosphodiesterase type 5 inhibitors for erectile dysfunction. Nat Clin Pract Urol. 2005;2:239-247.

9. Lue TF. A patient's goal directed approach to erectile dysfunction and Peyronie's disease. Can J Urol. 1995;2(S1):3-17.

10. Montague DK, Jarow JP, Broderick GA, Dmochowski RR, Heaton JP, Lue TF, et al. Chapter 1: The management of erectile dysfunction: an AUA update. J Urol. 2005;174(1):230-239.

11. Hatzimouratidis K, Hatzichristou DG. A comparative review of the options for treatment of erectile dysfunction: which treatment for which patient? Drugs. 2005;65(12):1621-1650.

12. Lue TF. Erectile dysfunction. N Engl J Med. 2000;342:1802-1813.

13. Rosen RC, Kostis JB. Overview of phosphodiesterase 5 inhibitors in erectile dysfunction [abstract]. Am J Cardiol. 2003;92:9-18M.

14. Stroberg P, Hedelin H, Ljunggren C. Prescribing all phosphodiesterase 5 inhibitors to a patient with erectile dysfunction - a realistic and feasible option in everyday clinical practice - outcomes of a simple treatment regime. Eur Urol. 2006;49:900-907.

15. Wright PJ. Comparison of phosphodiesterase type 5 (PDE5) inhibitors. Int J Clin Pract. 2006;60(8):967-975.

16. Gresser U, Gleiter $\mathrm{CH}$. Comparison of efficacy and side effects of the PDE-5 inhibitors sildenafil, vardenafil and tadalafil. Eur J Med Res. 2002;7:435-446.

17. Porst H, 2006. Oral pharmacotherapy of erectile dysfunction. In Standard practice in sexual medicine. 1st ed. Blackwell Publishing; 2006. p 83-86. 
18. Sommer F, Engelmann U. Future options for combination therapy in the management of erectile dysfunction in older men. Drugs Aging. 2004;21:555-564.

19. McCullough AR, Barada JH, Fawzy A, et al. Achieving treatment optimization with sildenafil citrate (Viagra) in patients with erectile dysfunction. Urology. 2002;60(2 Suppl 2):28-38.

20. McMahon C. High dose sildenafil citrate as a salvage therapy for erectile dysfunction [abstract]. Int J Impotence Res. 2002;14 Suppl 3: S89, Abstr, P5.11.

21. McMahon C. Efficacy and safety of daily tadalafil in men with erectile dysfunction previously unresponsive to on-demand tadalafil. J Sex Med. 2004;1:292-300.

22. Porst H. Salvagetherapie mit taglicher dosierung von sildenafil bei nonrespondern auf on-demand therapie mit $100 \mathrm{mg}$ sildenafil. Urologe A. 2003;42(10):1330-1336.

23. Cartledge J, Eardley J, orchard C, et al. Impairment of corpus cavernosal smooth muscle relaxation by glycosylated haemoglobin [abstract]. Eur Urol. 1999;35 Suppl 2 :100, Abstr. 399.

24. Saltzman EA, Guay AT, Jacobson J. Improvement in erectile function in men with organic erectile dysfunction by correction of elevated cholesterol levels: a clinical observation. J Urol. 2004;172:255-258.

25. Morales A, Buvat J, Gooren LJ, et al. Endocrine aspects of sexual dysfunction in men. $J$ Sex Med. 2004;1:69-81.

26. DeBusk R, Drory Y, Goldstein I, et al. Management of sexual dysfunction in patients with cardiovascular disease: recommendations of the Princeton consensus panel. AmJ Cardiol. 2000;86(2):175-181.

27. Jackosn G, Martin E, McGing E, et al. Successful withdrawal oral longacting nitrates to facilitate PDE5 inhibitor use in stable coronary disease patients with erectile dysfunction. J Sex Med. 2005;2:513-516.

28. Viagra (sildenafil citrate) Prescribing Information. Accessed March 2008. Available from http://www.pfizer.com/files/products/uspi_viagra.pdf.

29. Levitra (vardenafil $\mathrm{HCl}$ ). Prescribing information. Accessed March 2008. Available from http://www.univgraph.com/bayer/inserts/ levitra.pdf.
30. Cialis (tadalafil). Prescribing information. Accessed March 2008. Available from http://pi.lilly.com/us/cialis-pi.pdf

31. Kloner RA, Jackson G, Emmick JT, et al. Interaction between the phosphodiesterase 5 inhibitor, tadalafil and 2 alpha-blockers, doxazosin and tamsulosin in healthy normotensive men. $J$ Urol. 2004;172:1935-1940.

32. Hedelin H, Stroberg P. Treatments for erectile dysfunction based on patient-reported outcomes. Drugs. 2005;65:2245-2251.

33. Von Keitz A, Rajfer J, Segal S, et al. A multicenter, randomized, doubleblind, crossover study to evaluate patient preference for tadalafil and sildenafil. Eur Urol. 2004;45:499-507.

34. Stroberg P, Murphy A, Costigan T. Switching patients with erectile dysfunction from sildenafil citrate to tadalafil: results of a European multicenter, open-label study of patient preference. Clin Ther. 2003;25:2724-2737.

35. McMahon CM, Kozlowski RK, Kaufman AK, et al. Potential predictors for treatment preference in men with erectile dysfunction taking sildenafil and tadalafil in an open-label switch trial [abstract]. $J$ Sex Med. 2004;1 Suppl 1:40.

36. Eardley I, Mirone V, Montorsi F, et al. An open-label, multicentre, randomized, crossover study comparing sildenafil citrate and tadalafil for treating erectile dysfunction in men naive to phosphodiesterase 5 inhibitor therapy. BJU Int. 2005;96:1323-1332.

37. Claes HIM, Van Poppel H. The use of sildenafil, tadalafil and vardenafil in clinical practice [abstract]. J Sex Med. 2004;1 Suppl 1:42.

38. Mulhall JP, McLaughlin TP, Harnett JP, Scott B, Burhani S, Russell D. Medication utilization behavior in patients receiving phosphodiesterase type 5 inhibitors for erectile dysfunction. $J$ Sex Med. 2005;2:848-855.

39. Kell P, Hvidsten K, Morant S, et al. Factors that predict changing the type of phosphodiesterase type 5 inhibitor medication among men in the UK. BJU Int. 2007;99:860-863.

40. Mulhall JP, Montorsi F. Evaluating preference trials of oral phosphodiesterase 5 inhibitors for erectile dysfunction. Eur Urol. 2006;49:30-37. 